\title{
Conoscere per formulare e comunicare le strategie: il ruolo del business plan
}

\author{
GIOVANNA MARIANI
}

\begin{abstract}
Obiettivi: La finalità del lavoro è quello di rilevare i knowledge drivers che il business plan deve contenere secondo l'ottica del redattore e dell'investitore per supportare la valutazione della fattibilità dell'operazione e l'analisi del livello del rischio, nel caso di M\&A e di operazioni di turnaround.

Metodologia: Le tesi sono argomentate con un'analisi della bibliografia, completate con considerazioni emerse da interviste rivolte alle due tipologie di attori.

Risultati: I risultati mostrano che il business plan costituisce ancora un basilare strumento di conoscenza per supportare una consapevole formulazione delle strategie e per trasmettere tale conoscenza, comunicando appunto le strategie agli stakeholders, soprattutto in un'ottica di allineamento informativo tra il redattore ed il finanziatore.

Limiti della ricerca: Le considerazioni che emergono potrebbero trovare ulteriore supporto di informazioni e di significatività con un'indagine empirica svolta su altre operazioni e ad altri attori interessati al processo di business planning.

Implicazioni pratiche: Si potrebbe osservare che il maggior contributo del lavoro è proprio sul piano pratico, in quanto presenta una metodologia operativa.

Originalità del lavoro: La tematica in oggetto presenta indubbiamente un ampio sviluppo in letteratura. Si può, comunque, rilevare una certa originalità proprio sul piano del contributo operativo, ma anche in merito al rilievo strategico.

Parole chiave: business plan; rischio; $M \& A$; turnaround management; private equity

Purpose of the paper: The aim of this study is to detect knowledge drivers that business plan should include in the editor's and the investor's perspectives to assess the feasibility of the operation and risk level in $M \& A$ and turnaround activity.

Methodology: Theses are argued with an analysis of the literature with the support of considerations of some interviews with the two types of actors.

Findings: The results show that business plan is still a tool of knowledge to support a conscious strategies formulation and to get to stakeholders across, with particular attention to alignment information between the editor and investor.

Research limits: Limitations of the research and considerations that emerge may find additional support information and significance with an empirical study carried out on other operations and other stakeholders in the process of business planning.
\end{abstract}

* Ricercatore Confermato di Finanza Aziendale- Università degli Studi di Pisa e-mailgmariani@ec.unipi.it 
Practical implications: It could be argued that the major contribution of the work is just on a practical level, because it presents a methodology.

Originality of the work: The issue in question has undoubtedly extensive literature. You can still detect a certain originality in terms of its contribution to operational and on strategic role.

Key words: business plan; risk management; $M \& A$; turnaround management; private equity

\section{Sintesi della letteratura e domande di ricerca}

Il primo dubbio che può assalire, legittimamente, un lettore che dovesse incuriosirsi a tale lavoro è: cosa si potrà mai dire di nuovo sul business plan? Solo digitando il termine business plan su qualsiasi motore di ricerca si corre il rischio di spaventarsi dai riferimenti, di varia natura, che è possibile consultare a tale riguardo. Eppure, nonostante ciò, il dilemma, il dibattito, sull'utilità o no di tale strumento di pianificazione è sempre alquanto vivace, soprattutto sul piano internazionale, sia tra gli scholars che tra i practitioners. La questione sul to be or not to be del business plan ha decisamente creato una spaccatura tra i sostenitori e coloro che, invece, ritengono che esso abbia oramai fatto il suo tempo e che debba essere soppiantato da nuovi modelli di pianificazione strategica, più sofisticati sul piano quantitativo ${ }^{1}$, che siano in grado di catturare il più ampio ventaglio possibile di variabili, ma che soprattutto siano atti a gestire, in modo predittivo, l'elevata incertezza del mercato (Kuehn et al., 2009).

Ad oggi, dunque, la letteratura si divide tra coloro che non riconoscono alcuna utilità del business plan per l'imprenditore (The learning school), e quelli che, invece, ne promuovono ancora la sua validità (The planning school), anzi investendolo di una valenza strategica, soprattutto in fasi critiche del ciclo di vita dell'impresa (Borges et al., 2013). Secondo i primi (tra cui Bewayo, 2010; Brinckmann et al., 2010; Lewitt e March, 1988; Sahalmn, 2007; Timmons e Spinelli, 2007), il business plan ingesserebbe l'iniziativa, la genialità, l'inventiva dell'imprenditore, il quale per redigere il progetto è distolto dai suoi fondamentali compiti: ricercare nuovi clienti, progettare un'efficace supply chain e, soprattutto se trattasi di scienziato-imprenditore, progettare nuovi prodotti. Il gap culturale in termini di business management che, in genere, contraddistingue un imprenditore di formazione scientifica renderebbe il progetto impegnativo ed inutile, time consuming e quindi costoso (Lee, 2000).

Il business plan, visto strumentale solo ad una funzione di fund raising, può risultare un mero assemblaggio di assumptions, sottoposte poi ad un arduo processo di stima e quindi con un elevato margine di errore (Sahalmn, 2007; Timmons e Spinelli, 2007). Honig e Karlsson (2001, 2004), hanno difatti osservato che molti

1 Nell'ambito della pianificazione in condizioni d'incertezza si stanno sviluppando numerosi modelli di programmazione lineare stocastica. Si vedano, tra gli altri: Brandimarte, 2006, e Aliprantis e Chakrabarti, 2000. 
imprenditori redigono il business plan "...because they feel obligated to do so". L'imprenditore indotto alla pianificazione, soprattutto per legittimare il suo business, è portato a fornire un'immagine positiva che potrebbe indurre un atteggiamento di "superstitious learning". Lewitt e March (1988) sostengono che il promotore dell'iniziativa potrebbe a sua volta convincersi di certi scenari ("emotional involvement", Gumbert, 2002), e assumere comportamenti dannosi, con risultati deludenti.

Sul piano opposto si pone The planning school (Armstrong, 1982; Karlsoon e Honig, 2009; Bewaio, 2010), in cui si riconosce al business plan una duplice valenza strategica. Il team imprenditoriale riesce ad "immagazzinare" conoscenza per supportare una consapevole formulazione delle strategie e trasmettere tale conoscenza, comunicando appunto le strategie agli stakeholders, nel senso ampio del termine, per coinvolgerli attivamente nella realizzazione delle stesse. Occorre ricordare a tale proposito che le economie moderne si stanno rapidamente evolvendo verso schemi learning economies (OECD, 1996), in cui s'impone la visione knowledge-based dell'impresa (Pinch et al., 2003). Secondo tale orientamento la competitività di lungo periodo di un'impresa è fortemente condizionata dalla sua abilità di creare conoscenza, di innovare i suoi processi produttivi e di apprendere continuamente. I leader sono quelle imprese che investono maggiori risorse nei processi di ricerca, di apprendimento delle nuove tecnologie e nell'innovazione. La conoscenza, un processo o struttura dinamica attraverso cui l'informazione può essere "stored, processed and understood" (Howells, 2002), diventa quindi un asset complesso e multidimensionale la cui diffusione richiede articolati processi cognitivi. Il business plan rappresenta, quindi, un asset per l'impresa in quanto esso consente di supportare un processo di autovalutazione ai fini decisionali, soprattutto in fase di costituzione e nelle operazioni strategiche (M\&A e Turnaround), di sviluppare un sistema di programmazione e di controllo delle attività nonché di condivisione delle scelte con gli altri players: il tutto per supportare una consapevole formulazione e sviluppo delle strategie (Schaefer, 2011). Nel Piano l'imprenditore ha la possibilità di "edificare" in modo dettagliato l'intera operazione. L'obiettivo principale, quindi, è quello di giungere ad una conoscenza approfondita di tutte le problematiche che investono l'impresa a $360^{\circ}$, di definire, altresì, un quadro abbastanza completo di tutti i rischi e le incertezze connesse all'iniziativa, ma soprattutto di perfezionare un processo cognitivo per la valutazione della concreta fattibilità del progetto (Sarasvathy, 2001). Il business plan, inoltre, in fase di realizzazione del progetto diventa un "segnalatore di direzione", una bussola per lo svolgimento delle attività, fornendo una base per lo scheduling di tutte le singole operazioni. Con esso è possibile ricostruire una roadmap delle attività da sottoporre poi a confronto con i dati a consuntivo che progressivamente si perfezionano, per rilevare eventuali gaps, sulla base dei quali rivedere le assumptions formulate e ipotizzare nuovi scenari (Gendrom, 2004; Ferrandina e Carriero, 2010; Ford et al., 2008; O’Connor, 1998). Il business plan, difatti, non deve essere visto come un modello da compilare che esprime uno status quo, ma esso richiede un "atteggiamento processuale" di una realtà in divenire. (Correale e Penco, 2005; 
Mariani, 2012a). Il Piano è un processo dinamico (Thomsen, 2009), che ha proprio il compito di stimolare il redattore (il team imprenditoriale, il management, etc.) a rilevare la necessità di "cambiare rotta", di riformulare scelte strategiche, di rivedere le assunzioni, quando l'iniziativa è ancora sulla carta. Il business planning può svolgere un ruolo aggregante e motivazionale, esso, difatti, comporta l'esame di tutti gli aspetti gestionali, anche di quelli che potrebbero apparire non influenzabili direttamente dal progetto; con esso tra l'altro, si mira alla condivisione delle scelte da parte di chi in azienda vive (Baccarani, 2009), a diffondere un concreto senso di motivazione e fiducia. Quando il business plan è riconosciuto come strumento di guida gestionale, le imprese ne traggono effetti positivi sul processo di creazione di valore (Lumpkin et al., 1998).

Un'indicazione di tale importanza è data dal fatto che, seppur in modo approssimato, ogni anno sono redatti circa 10 milioni di business plan nel mondo (Gumpert, 2002). Anche in contesti economici più evoluti, come appunto quello statunitense, è stata evidenziata una certa correlazione tra l'uso a redigere il business plan ed il tasso di fallimento delle piccole unità: le minori imprese che hanno sviluppato una più consolidata cultura a tale metodologia di pianificazione hanno rilevato migliori risultati (Perry, 2001). Esso gioverebbe al coordinamento interno e al controllo delle attività riducendo il tasso di fallimento (Karlosoon e Honig, 2009). Dopo decenni di stimolo culturale su tale metodologia, molti programmi accademici e professionali di formazione imprenditoriale (Katz, 2007, Baum et al., 2007, Kuehn et al., 2009) contemplano, difatti, l'insegnamento del business plan process e normalmente trovano come momento conclusivo proprio la stesura di un piano imprenditoriale, al punto che Katz e Green (2007) parlano di ubiquitous business plan. In tale direzione diverse ricerche (Gendron, 2004; Karlsoon e Honig 2009), sottolineano il fatto che il mondo imprenditoriale ha ormai metabolizzato l'assioma che il business plan debba essere riconosciuto come basilare momento di autovalutazione e come strumento di comunicazione che consente una legitimacy (Karlsoon e Honig, 2009) all'esterno del business. La sua funzione di strumento comunicativo, soprattutto con $\mathrm{i}$ finanziatori, ha rappresentato la motivazione prevalente della sua nascita, diffusione e del successo raggiunto all'interno del sistema economico internazionale.

Una delle valenze strategiche del business plan è proprio quella di favorire il processo cognitivo esterno degli stakeholders per coinvolgerli attivamente nell'operazione oggetto di analisi (Hormozi et al., 2002), per contribuire a creare fiducia negli interlocutori esterni al progetto (Baccarani, 2009). Esso costituisce, indubbiamente, il documento centrale mediante il quale l'imprenditore è in grado di rendere chiare e leggibili idee che altrimenti potrebbero rimanere vaghe. Passare dalle idee alla definizione del fabbisogno finanziario, alla quantificazione dei probabili ritorni finanziari e della successione temporale degli stessi (Carlesi, 1999), costituisce un primo "biglietto da visita" per la comunità finanziaria (Lee, 2000), per la quale diventa, inoltre, basilare la valutazione della capacità di credito dell'impresa sul medio-lungo termine. La ricerca dei finanziamenti da parte delle imprese di ogni dimensione necessita sempre più della presentazione di un business plan, sia per $\mathrm{i}$ 
finanziatori di private equity (Anderson, 2000), per i quali costituisce un must, ma anche nei rapporti con il mondo bancario (Bruns, 2004). Considerando l'ampio ventaglio di attori interni ed esterni alla compagine aziendale interessati al contributo conoscitivo del business plan diventa legittimo porsi il quesito su quali debbano essere le informazioni basilari e puntuali da produrre e da trasmettere affinché si possa soddisfare una delle finalità del processo di planning: la conoscenza per la comunicazione. Pur rilevando che il business plan sia tradizionalmente associato ad una situazione di start-up, esso può svolgere un basilare contributo come knowledge driver in ogni attività lungo il ciclo di vita dell'impresa (Mariani, 2012a). Passando, infatti, dal tradizionale business plan in fase di start up (spin off), esso costituisce un processo basilare in un'impresa going concern per la valutazione di una nuova iniziativa, per il lancio di un nuovo prodotto, per la diversificazione di mercati geografici, per giungere, poi, al suo basilare contributo nelle operazioni di corporate finance, come nelle M\&A, nelle IPO, nel turnaround, nei processi di ristrutturazione, nelle partnership industriali e commerciali di una certa portata.

L'obiettivo del presente articolo si inserisce in questo ampio dibattito della dottrina e focalizza l'analisi sulle specificità del processo di planning in due delle operazioni di maggiore criticità delle imprese going concern, quali l'expansion e il turnaround. Si tratta di operazioni complesse, di natura straordinaria, che generano fabbisogno finanziario elevato, dove il coinvolgimento sia dei finanziatori di debt che di equity, ma anche di tutti gli altri stakeholders, può risultare consistente e determinante.

Occorre, inoltre, sottolineare il fatto che esse possono produrre effetti di ampia portata sul processo di creazione di valore (Bigelli e Mengoli, 1999) e sul livello di rischio, sia in termini positivi ma anche negativi, ma soprattutto in merito al rischio di default (Cartwright e Schoenberg; 2006), in particolare in un fase di environmental jolt come quella attuale (Wan e Yiu; 2009).

L'analisi del business plan diventa, difatti, una delle operazioni fondamentali nell'ambito dell'attività dei fondi di equity per valutare, sulla base anche degli elementi qualitativi, le opportunità di un'operazione, nonché il profilo di rischiorendimento. Per essi, quindi, poter derivare informazioni sulla fattibilità dell'operazione, in termini economico-finanziari, come tracciare le implicazioni gestionali che ne possono scaturire e gli effetti sul livello di rischio della stessa, diventa decisivo sia per la fase decisionale che per quella di comunicazione (Borello, 2009; Ferrandina e Carriero, 2010; Ford et al., 2008; Stutely, 2008; Parolini, 2011). Le principali domande di ricerca cui l'articolo vuole dare risposta possono essere declinate come di seguito indicato.

1. Quali sono le informazioni utili per la valutazione della fattibilità dell'operazione? Quali sono i driver conoscitivi utili sia nell'ottica del redattore che del valutatore esterno?

2. Quali sono le informazioni utili per la valutazione del livello di rischio esprimibile dall'operazione? Con quali driver conoscitivi è possibile per il valutatore tracciare un'ipotesi di rischiosità del business? 
Lo schema del lavoro prevede una prima parte che riflette su alcuni capisaldi consolidati nella letteratura sul processo di business planning e analizza la metodologia della ricerca. Di seguito si focalizza l'analisi sulle specificità conoscitive che rispettivamente il redattore ed il valutatore esterno, quest'ultimo rappresentato dai finanziatori di equity, ritengono utili nel business plan per le operazioni di M\&A, in termini di analisi della fattibilità e del livello del rischio. Nella terza parte l'ottica si sposta sulle operazioni di turnaround rispondendo ai due quesiti di cui sopra.

Le considerazioni che seguono tengono anche conto di interviste svolte con practitioners, sia in ottica di investitore/valutatore che di redattore del documento, $\mathrm{i}$ quali ci hanno consentito di aggiungere aspetti operativi, informativi e comunicativi rilevanti.

Le conclusioni e i possibili spunti futuri di ricerca sono a chiusura dell'articolo.

\section{Metodologia di ricerca}

Nelle parti precedenti si è avuto modo di osservare che il business plan rappresenta un momento basilare in cui la conoscenza per la formulazione delle strategie da parte del team imprenditoriale si fonde con la necessità di condivisione delle stesse con un ampio portafoglio di players. Diventa, quindi, estremamente critico, per il redattore del documento, avere la consapevolezza di quali possano essere i knowledge drivers per la valutazione della fattibilità e del rischio che il destinatario del documento si attende di trovare per il processo di valutazione. Il business plan può risultare, difatti, fortemente condizionato dall'obiettivo per cui esso è predisposto e dall'interlocutore cui è rivolto: un vivace dibattito, spesso avvolto da luci ed ombre, si è, difatti, progressivamente sviluppato proprio sugli elementi conoscitivi basilari su cui gli operatori di venture capital impostano la propria valutazione.

Il know-how che sostiene il complesso processo di analisi e valutazione costituisce spesso l'elemento di forza del successo di tali operatori, che quindi spesso non amano condividere.

Ai fini di poter delineare uno schema degli elementi conoscitivi principali che il business plan deve esprimere è stata promossa un'indagine qualitativa, condotta mediante interviste dirette a professionisti con ampia esperienza dal lato della redazione e/o valutazione del Piano di fattibilità.

Per ciascuna delle fattispecie in analisi (M\&A e Turnaround) sono stati coinvolti due professionisti che da tempo svolgono attività di consulenza sulle tematiche in oggetto, che vantano nel proprio "portafoglio" un numero elevato di operazioni condotte e che si siano resi disponibili a confrontarsi su tutti gli aspetti che investono il loro processo decisionale/investigativo, anche su quelle "strategiche". Ad ogni interlocutore è stata inviata in precedenza una check-list sugli argomenti da approfondire, articolata sostanzialmente in quattro sezioni: elementi di base del business plan, i knowledge driver per l'analisi della Fattibilità dell'operazione e del 
livello di rischio, Informazioni aggiuntive. Sono state poi condotte diverse interviste dirette ad ognuno di essi, in parte telefoniche e in parte personalmente, proprio per riuscire a ricostruire il complesso e delicato processo di analisi e di valutazione, i parametri di natura quantitativa, ma anche in merito alle considerazioni strategico/qualitative che sottendono la complessa valutazione.

Per quello che attiene la figura dell'Investitore/Valutatore sono stati intervistati il Dott. Luciano Anzanello, di Atlantis Partners, ed il Dott. Ottavio Conigliaro, di I2 Capital Partners SpA.

Relativamente al ruolo del redattore sono stati intervistati il Dott. Franco Abbate del Gruppo Twice - Medinvest S.p. $A^{2}$ e la Dott.ssa Alessandra Bechi dell'Associazione Italiana del Private equity e Venture Capital (AIFI), la quale ha fornito il suo importante supporto informativo sui contenuti del Business plan in ottica di strumento di accesso al mercato del private equity. Il Dott. Luciano Anzanello, ha contribuito anche in ottica di Redattore, in quanto, come operatore di uno dei più importanti turnaround fund può essere coinvolto anche nel ruolo di redazione del Piano.

\section{La valutazione nelle operazioni di M\&A: la fattibilità ed il livello di rischio}

In ambito finanziario, con il termine M\&A si individua un'ampia gamma di attività, di natura anche molto diversa tra loro, che hanno come comune denominatore l'obiettivo di accrescere il valore di uno o più business già esistenti (Arnold, 2013; Conca, 2010; Datta et al., 2013; Sadarsanam, 2003), talvolta promosse per la sopravvivenza dell'impresa (DePamphilis, 2012). Le acquisizioni sono un fenomeno economico di ampie dimensioni che richiedono mercati finanziari dinamici e risentono fortemente delle fasi congiunturali, presentando trend fluttuanti in corrispondenza degli andamenti del sistema economico (Martynova e Renneboog, 2006).

Di particolare interesse, difatti, sono i dati sul volume delle operazioni nel periodo 2009-2010, in cui le M\&A hanno segnato una forte contrazione dovuta, appunto, alla Crisi Finanziaria Globale, raggiungendo un minimo storico nel 2012 (KPMG, 2013; Wan e Yiu, 2009; Netter et al., 2010). Gli ambiti di ricerca sulle corporate acquisitions sono molteplici e cross culture, coinvolgendo gli aspetti squisitamente strategici, quelli procedurali fino ad investire gli effetti in termini di creazione o distruzione di valore (King et al., 2004; Laabs e Schiereck, 2010,

2 I2 Capital Partners SpA. è una SGR, facente capo alla Intek, che dal luglio del 2007 ha assunto la veste di fondo di private equity vero e proprio. Il fondo investe in special situations, sia operating, che non operating. La Atlantis Capital Special Situations S.p.A. è il primo investitore istituzionale indipendente dedicato alle imprese italiane in "situazioni speciali". Medinvest, al momento dell'indagine, rappresentava un gruppo internazionale specializzato in attività di "merchant banking". 
Kwoka e Pollitt, 2010, Gomes et al., 2013). Un'acquisizione, difatti, può aiutare un'impresa a raggiungere rapide posizioni competitive sul mercato e a garantirsi particolari vantaggi sinergici dalla combinazione con altre unità (Deutsch et al., 2011). Diverse ricerche hanno evidenziato a tale proposito, però, risultati contraddittori: le M\&A possono produrre effetti positivi sul valore di mercato delle imprese target, ma generare sostanziali perdite sul piano contabile e un elevato tasso di fallimento delle operazioni (Agrawal e Jaffe, 2000; Cartwright e Schoenberg, 2006). Tra le cause di fallimento di operazioni di M\&A, riportate in letteratura, emergono gli errori di valutazione del management, il quale tenderebbe a sovrastimare le sinergie e a sottostimare i costi di integrazione, e la presenza di un piano strategico carente o inappropriato (Marafioti, 2005).

La predisposizione del business plan riveste, quindi, un ruolo cardine nell'ambito della conoscenza interna e della comunicazione all'esterno dei piani di sviluppo dell'azienda e delle performance attese, in termini di flussi reddituali e di cassa. Si tratta, indubbiamente, di progetti in cui la stesura di un Piano da parte dell'impresa acquirente (bidder) è alquanto diffusa, sia per l'analisi strategica ai fini decisionali che come strumento di comunicazione con gli stakeholders.

Nelle operazioni di M\&A l'impresa bidder è chiamata a realizzare un doppio processo di valutazione, uno di tipo interno e l'altro di ottica esterna. Nel primo caso, l'analisi di un ampio ventaglio di informazioni ha come obiettivo conoscitivo di sintesi la quantificazione del livello di fabbisogno finanziario necessario per sostenere l'operazione, la valutazione dell'impatto in termini di creazione di valore, gli effetti in termini di risk management nonché la "compatibilità finanziaria": in sostanza, la valutazione della fattibilità complessiva del progetto, secondo quattro livelli: fattibilità imprenditoriale, fattibilità sul piano esterno, fattibilità sul piano interno e fattibilità economico-finanziaria e patrimoniale (Mariani, 2012a), (tab. 1).

Il quesito cui deve rispondere l'analisi della Fattibilità sul piano imprenditoriale può essere così sintetizzato: dispone l'impresa del know-how, il management ha messo in gioco la governance, gli skills adeguati per poter "edificare" il nuovo progetto? Qual è la qualità del team imprenditoriale e dei collaboratori? (tab. 1).

Soprattutto il valutatore esterno, difatti, prima di intraprendere una determinata operazione, si aspetta che dal documento emerga chiaramente il fatto che il management della bidder abbia svolto un articolato e distaccato processo di autovalutazione focalizzato, appunto, a rilevare i punti di forza e di debolezza, nonché le competenze chiave da mettere in gioco, affinché in fase di esecuzione del progetto non si manifestino devastanti vacatio gestionali che potrebbero vanificare $\mathrm{i}$ risultati, a causa di comportamenti improvvisati e impreparati. La presenza di competenze, di motivazione, dell'aligment tra i differenti attori e la business strategy costituisce per l'investitore un elemento basilare ai fini della valutazione della fattibilità. Dall'analisi della tab. 1 emerge, invece, un disallineamento di vedute tra $\mathrm{i}$ due players, in quanto il redattore del business plan considera quasi scontato tale aspetto, non ritiene opportuno approfondimenti ad hoc, sottovalutandone la rilevanza conoscitiva per l'interlocutore. 
Tab. 1: Knowledge drivers in M\&A operations

\begin{tabular}{|c|c|c|}
\hline \multirow[t]{2}{*}{ a)FATTIBILITA' } & REDATTORE & INVESTITORENVALUTATORE \\
\hline & $\begin{array}{l}\text { 1- Fattibilità sul piano esterno } \\
\text { - Mercato, Settore } \\
\text { 2- Fattibilità econ-fin. } \\
\text { - Analisi azienda target } \\
\text { - Opportunità di crescita-sinergie } \\
\text { di costo, ricavi, fiscali, finanziarie } \\
\text { - Marginalità, } \\
\text { - Indebitamento } \\
\text { - Prezzo di acquisto, rapporto di } \\
\text { concambio, opportunità di } \\
\text { crescita } \\
\text { - Valore Capitale economico stand } \\
\text { - Wane ex ante e ex post } \\
\text { - Tacc } \\
\text { Timing analisi cash flow su base } \\
\text { Coerenzale } \\
\text { Con assumptions }\end{array}$ & 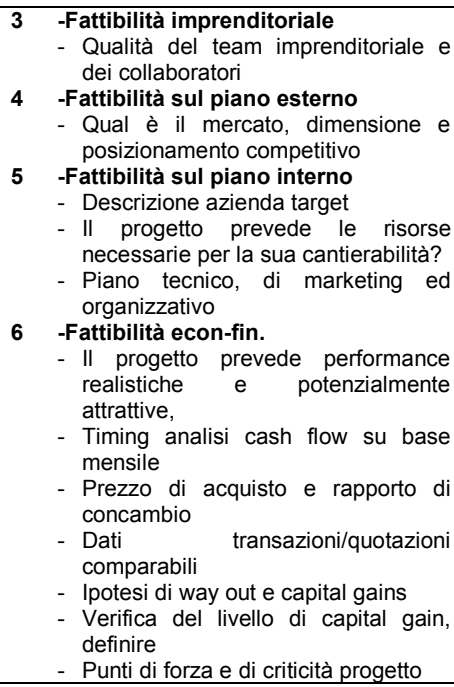 \\
\hline \multicolumn{3}{|l|}{ a) b) Risk analysis } \\
\hline Rischio di mercato & $\begin{array}{l}\text { 1) } \text {-Struttura del Beta } \\
\text { - Criticità delle barriere all'entrata } \\
\text { - } \text { Tasso di crescita } \\
\text { - Concentrazione concorrenza } \\
\text { - Quota di mercato gestita } \\
\text { - Fattori di vantaggio competitivo } \\
\text { - Dimensione dell'impresa }\end{array}$ & $\begin{array}{l}\text { 4) Criticità delle barriere all'entrata } \\
\text { - Tasso di crescita } \\
\text { - Concentrazione concorrenza } \\
\text { - Grado di regolamentazione } \\
\text { pendenze legali } \\
\text { - Struttura dei costi } \\
\text { - Quota di mercato gestita } \\
\text { - Fattori di vantaggio competitivo } \\
\text { - Dimensione dell'impresa } \\
\end{array}$ \\
\hline $\begin{array}{l}\text { Rischio finanziario ed } \\
\text { economico }\end{array}$ & $\begin{array}{ll}\text { 2) } & \text {-Indici di indebitamento } \\
\text { - } & \text { Quozienti di tesoreria } \\
\text { - } & \text { Quoziente di disponibilità } \\
\text { - } & \text { Leva finanziaria } \\
\text { - } & \text { Free Operating Cash } \\
& \text { Flow/Debito } \\
\text { - } & \text { Indici di rotazione del capitale } \\
& \text { circolante } \\
\text { - } & \text { Ebitda/Vendite } \\
\text { - } & \text { Ros, Roi } \\
\text { - } & \text { Investimenti aggiuntivi (CapEx) } \\
\end{array}$ & $\begin{array}{l}\text { 5) Indici d'indebitamento } \\
\text { - Leva finanziaria } \\
\text { - Free Operating Cash Flow /Debito } \\
\text { - Leva operativa } \\
\text { - Indici di rotazione del capitale } \\
\text { circolante } \\
\text { - Ebitda/Vendite } \\
\text { - CapEx/EBITDA } \\
\text { - Analisi di previsione del rischio di } \\
\text { default }\end{array}$ \\
\hline Rischio di progetto & $\begin{array}{l}\text { 3) Struttura finanziaria ex-post, NPV, } \\
\text { IRR, BEP }\end{array}$ & $\begin{array}{l}\text { 6) Struttura finanziaria ex-post, IRR, BEP, } \\
\text { Multipli del capitale investito }\end{array}$ \\
\hline
\end{tabular}

Fonte: ns. elaborazione

L'osservazione si estende anche alla Fattibilità sul piano interno (tab. 1) che l'investitore considera di rilievo strategico per la "conoscenza" del business, mentre il redattore ritiene sia pacifico che l'impresa bidder sia in grado di gestire il processo d'integrazione dal punto di vista operativo. L'investitore, d'altro canto, è interessato a conoscere quali siano i piani, tecnico, di marketing ed organizzativo, che il management dell'impresa bidder pensa di attuare per il successo e monitoraggio 
dell'operazione. Parte strategica di questa fase di analisi, per entrambe i players, è lo status quo della target, che in un primo stato esplorativo avviene unicamente sulle informazioni disponibili sul mercato, ma che dovranno progressivamente essere integrate durante la trattativa, fino a giungere ad un completo approfondimento con la due diligence. L'obiettivo conoscitivo è quello di delineare le opportunità e i rischi che potrebbero essere generati dall'integrazione. Occorre richiamare l'attenzione sul fatto che sono proprio le difficoltà d'integrazione a rappresentare uno dei principali punti di criticità nel processo di creazione di valore nelle M\&A (Weber et al., 2012). In sostanza, nel piano il valutatore si attende di poter rilevare informazioni in merito anche ai numeri storici di un'azienda, in quanto il passato è un indicatore del futuro: il finanziatore di equity, infatti, cerca di valutare la "ragionevolezza delle assunzioni di un trend di crescita cercando conferme, o smentite, proprio dal passato" (Piana, 2012). Dallo schema si può osservare che entrambi gli attori ritengono strategiche le informazioni in merito alla Fattibilità sul piano esterno e quelle di natura economico-finanziaria, aspetti su cui si focalizza, in genere, l'attenzione del team di redazione. Le analisi del mercato, Fattibilità sul piano esterno (tab. 1), devono essere alquanto dettagliate per quello che attiene il settore, ponendo in essere un benchmark con i principali competitors, con particolare attenzione alle leve competitive, agli eventuali elementi di criticità su cui agire, soprattutto se l'opzione strategica è di diversificazione delle attività. Si può, difatti, osservare che l'investitore considera decisiva l'analisi della situazione competitiva (tab. 1), soprattutto per un'accurata valutazione del rischio di mercato, come sarà puntualizzato nella parte specifica. Trattandosi di un investimento di natura complessa il punto di sintesi ai fini valutativi è la parte della Fattibilità economico-finanziaria, dove si incrociano gli interessi conoscitivi del redattore e del valutatore, pur con alcune specificità (tab. 1). Si può, difatti, osservare che il redattore pone l'attenzione sull'esame delle aree di complementarietà e di sovrapposizione delle unità aziendali coinvolte per la valorizzazione delle sinergie $\mathrm{e}$, al contempo, sulla quantificazione dei costi di integrazione, tutto proteso ad una logica di shareholder value (tab. 1). È necessario, infatti, individuare i value drivers influenzati dalle sinergie, dalla natura e la probabilità di realizzo delle stesse: questa fase richiede particolare attenzione e cura in quanto, il valutatore è consapevole che, spesso, come in precedenza evidenziato, il management della bidder tende a sovrastimare le sinergie o a sottostimare i costi o i tempi d'integrazione. Ai fini di valutazione interna si tratta, in sostanza, di raccogliere le informazioni che potranno essere inserite nel calcolo del Valore attuale Netto dell'acquisizione (NPV). Tra i fattori di criticità richiede un'elevata attenzione il timing dei cash-flows, per misurare la capacità di servizio al debito che l'impresa potrà garantire (tab. 1). In corrispondenza all'holding period di mercato si rileva un distinguo tra i due players: il management ritiene che sia sufficiente un orizzonte di pianificazione annuale, mentre l'investitore/valutatore esterno riconosce a tale elemento un maggior contenuto di rischiosità per cui richiede una tempificazione a livello mensile, soprattutto per il monitoraggio. Tali informazioni, congiuntamente all'analisi storica della società oggetto dell'operazione e del settore, sono essenziali per definire il 
valore stand alone dell'azienda target che, frequentemente, rappresenta la base di partenza per decidere la convenienza di un'operazione di M\&A, le ipotesi di prezzo e/o del rapporto di concambio, i tempi di intervento e le possibili modalità di finanziamento.

Dal lato dell'investitore, soprattutto di private equity, le finalità conoscitive sono, oltre a quelle sopra menzionate, anche rappresentate dalle informazioni sul livello di criticità dell'intera operazione, per cui nel business plan sono ricercate indicazioni sui punti di forza e di debolezza che l'impresa bidder potrebbe incontrare e dover risolvere. L'investitore tende, poi, a riformulare una propria ipotesi in termini di prezzo e/o di rapporto di concambio, nonché naturalmente a verificare l'exit strategy (IPO, trade sale, secondary market, etc.) ed il livello di capital gain potenzialmente realizzabile. Il business plan costituisce un'indubbia valenza conoscitiva anche per gli altri soggetti in qualche modo coinvolti nell'operazione, quali sindacati, fornitori, clienti, comunità finanziaria, soggetti pubblici, tutti gli stakeholders il cui ruolo attivo o, talvolta di opposizione, assunto nell'operazione può essere determinante per il costo e la concreta realizzazione di un'operazione di M\&A.

L'analisi del rischio di un'operazione rappresenta indubbiamente uno dei processi fondamentali nell'ambito della valutazione di un progetto da parte di un investitore di equity (tab. 1).

$\mathrm{Si}$ tratta di tradurre tutti gli elementi qualitativi, che fanno percepire un'opportunità come attraente $\mathrm{o}$ meno, in un insieme di aspettative quantitative razionali circa il futuro di un'azienda, per valutare soprattutto il profilo di rischiorendimento. Oltre all'impatto sui valori economici, finanziari e patrimoniali prospettici sopra menzionati, difatti, le operazioni d'integrazione hanno effetto anche sul profilo di rischio, in termini di rischio di mercato (variabilità dei ricavi), di rischio economico-finanziario (leva operativa e leva finanziaria), delle società coinvolte, nonché il rischio di progetto. Aspetto questo che costituisce un elemento di conoscenza determinante, soprattutto nell'ottica dell' investitore/valutatore (tab. 1). In merito al rischio di mercato si può individuare un sostanziale allineamento d'interesse conoscitivo tra i due players: si considerano espressivi i dati sul posizionamento competitivo e la quota di mercato che la nuova struttura potrà vantare, i fattori di vantaggio competitivo, le variabili relative alla dimensione dell'impresa, L'investitore è particolarmente interessato a verificare la presenza di eventuali spazi di perfezionamento di economie di scala e di barriere all'entrata di mercato, che potrebbero comportare impegnativi investimenti in fase di ingresso da un lato, ma garantire elevati margini alle imprese già presenti (tab. 1).

Il valutatore-finanziatore, ritiene, inoltre, che il business plan debba produrre informazioni anche sul grado di regolamentazione del settore, la presenza di pendenze legali e sulla struttura dei costi. Diventa, importante, difatti poter gestire una struttura dei costi elastica, in grado di fronteggiare eventuali "aggressioni" in termini di price competition. Le informazioni di natura quantitativa devono poi essere corredate da indicazioni di tipo qualitativo sulla presenza di possibili rischi che potrebbero annullare gli effetti sinergici, quali rischi di natura organizzativa e di 
tipo culturale, che nelle operazioni cross-border possono rappresentare i maggiori punti di criticità.

Sul rischio finanziario si concentra l'attenzione delle due ottiche di analisi, in quanto la percezione del livello di default arriva a produrre effetti importanti ed immediati sul costo del capitale, sul grado di affidamento e, comunque, sul processo di copertura del fabbisogno in generale. A tale proposito, soprattutto l'investitore (tab. 1-b)-5) potrebbe essere interessato ad un approfondimento sul rischio di default mediante un'analisi del rischio di insolvenza, attraverso i vari modelli predittivi, quali lo Z-score di Altman ed evoluzioni successive (Altman, 2000, Altman e Sabato 2007; Poddighe, 2009; Mariani, 2012b).

Il differenziale di rischio prodotto dall'operazione (Zanetti, 2000), sulle strutture coinvolte, rappresenta un elemento discriminante nella valutazione del NPV (tab. 1). Ai fini conoscitivi l'analisi s'incentra sul grado di indebitamento dell'azienda bidder, pre e post operazione, e sul costo del debito; questo emerge tipicamente dall'analisi per indici inclusa nel business plan. Come rilevabile in tab. 1 i due profili di analisi sono concordi nell'individuare alcuni indici basilari ai fini della valutazione. Tra questi si possono menzionare gli indici d'indebitamento e di gestione del circolante: Debiti/patrimonio netto e il Grado di leva finanziaria, di solito legati a covenant su finanziamenti bancari, il FOCF/debito ${ }^{3}$, a volte legato a meccanismi di rimborso obbligatorio su finanziamenti bancari, nonché tutti gli indici di rotazione del circolante e l'EBITDA/Vendite.

Pareri discordi sono, invece, stati espressi sugli indici di liquidità, di solvibilità e per il quoziente d'indebitamento finanziario, ritenuti basilari per il redattore ai fini del processo di autovalutazione, mentre il grado di leva operativa e il livello di Posizione Finanziaria netto sono considerati determinanti per l'investitore di private equity.

Per quanto attiene il rischio di progetto, attraverso il Piano si può evidenziare la relazione tra investimenti previsti e proiezioni in termini di crescita e marginalità dei flussi di cassa. Dovrà essere analizzata la struttura finanziaria post - acquisizione in quanto, nell'ottica del redattore (impresa acquirente), ad esempio, l'eccessivo indebitamento post operazione potrebbe creare danni al nuovo assetto, anche in caso di business redditizio (tab. 1). Per entrambi i professionisti, i metodi di valutazione finanziaria più idonei sono l'IRR ed il Break even point (BEP). L'IRR (Tasso Interno di Rendimento) visto soprattutto dall'investitore come parametro di mercato, anche se è frequente il ricorso a multipli sul capitale investito, con base Ebit. La valutazione attraverso il NPV, non è ritenuta necessaria per il finanziatore, mentre il redattore preferisce svilupparla a parte, non all'interno del Piano.

3 Si ricorda che FOCF è l'acronimo di Free Operating Cash Flow, con il quale rappresenta l'effettivo flusso monetario (cassa) dell'unità, tenuti in considerazione gli investimenti in capitale circolante e gli investimenti necessari all'operatività ed al mantenimento / accrescimento dell'attività nel lungo periodo. 


\section{II business plan nelle operazioni di turnaround: la fattibilità ed il livello del rischio}

Dopo anni in cui si è dibattuto su quali potessero essere i driver competitivi, si è preso coscienza che nel ciclo di vita dell'impresa si susseguono fasi positive di expansion e fasi negative. Il procrastinarsi nel tempo di una situazione negativa conduce però allo stato patologico che conosciamo con il nome di "crisi" e che si manifesta con pesanti squilibri di natura economica, patrimoniale e finanziaria, nonché organizzativa.

La questione che si pone è, in primo luogo, cosa debba intendersi per crisi, come possa essere prevista, misurata e, soprattutto, se e come essa possa essere risolta (Guatri, 1986). Si sono moltiplicati gli studi per la previsione della crisi (Altman 2000, Altman e Sabato, 2007, D’Annunzio e Falavigna, 2004, Hui e Jing-Jing, 2008, Lee e Yeh, 2004,) e per il suo superamento. Le modalità di soluzione della crisi sono molteplici e tra queste il turnaround, o risanamento, inteso come difesa e ricostruzione del valore dell'impresa e di un recupero sostenibile nel tempo della capacità di reddito aziendale, si trova davanti un mercato in crescita, nel numero di aziende in sofferenza, in insolvenza o in fallimento (Candelo, 2005, Mariani e Marsili, 2011; Mariani e Panaro, 2012).

Tale crescita ha registrato delle forti accelerazioni negli ultimi dieci anni, per effetto di fattori macroeconomici e per l'afflusso di capitali nel mercato del turnaround da parte di investitori specializzati, i volture funds, per i quali il processo conoscitivo ai fini valutativi diventa decisivo, con rilevanti implicazioni strategiche e sul rischio. La redazione del Piano di ristrutturazione/business plan, in questi casi, associa alla tradizionale funzione di strumento gestionale anche il ruolo di comunicazione del "cambiamento" verso tutti gli stakeholders coinvolti. Il Piano generalmente presuppone, a differenza delle altre fasi di vita dell'azienda, un intenso coinvolgimento anche dei portatori di interessi esterni all'azienda.

La struttura di un business plan/Piano di turnaround per un'operazione di turnaround (Candelo, 2005; Guatri, 1995) si rende necessario evidenziare alcuni aspetti salienti per la valutazione della fattibilità dell'operazione. Occorre precisare che la stessa nuova legge fallimentare ${ }^{4}$ nell'art. 67 richiede "un piano che appaia idoneo a consentire il risanamento della esposizione debitoria dell'impresa e ad assicurare il riequilibrio della sua situazione finanziaria". Il Piano di soluzione della crisi ha, quindi, una certa responsabilità in quanto deve "certificare" che sia possibile risanare l'impresa e che l'obiettivo sia fattibile e fondato su dati veritieri. Le target ideali, per un turnaround di successo, sono le imprese che possono vantare una posizione competitiva valida, con un brand e operanti in un mercato in

4 La nuova Legge Fallimentare, ispirata al Chapter 11 della legge fallimentare americana, ha spostato completamente la questione orientandosi alla creazione di un contesto favorevole al recupero di imprese in difficoltà ed al mantenimento della loro continuità, piuttosto che prevedere un inesorabile processo di smembramento, come disciplinato nella legislazione precedente. 
espansione. In considerazione di ciò sia il redattore che il valutatore hanno bisogno di effettuare valutazioni di tipo macroeconomico, in modo da rilevare i trend di settore, di mercato, la situazione concorrenziale, le opportunità di crescita che l'impresa in risanamento potrà cogliere.

Occorre precisare che in genere il Piano di turnaround è redatto dal personale interno all'azienda target, frequentemente supportato, però, da società di consulenza, vicine al fondo di turnaround, in grado di vagliare con maggiore lucidità i punti di debolezza e di forza. È rilevabile, a tale proposito, un allineamento di esigenze conoscitive tra le due visioni sulla Fattibilità gestionale e quella economico-finanziaria. In merito alla prima fattispecie, occorre individuare due fasi assolutamente peculiari che generalmente non si riscontrano in situazioni "in bonis" (Rojas-Arce et al., 2012).

Per il valutatore/investitore occorre, difatti, che siano analizzati i motivi che hanno portato al dissesto aziendale, sia esso solo finanziario o, nelle ipotesi peggiori, anche operativo (tab. 2). È necessario valutare attentamente se le cause della crisi sono temporanee, lasciando aperte quindi chance di riuscita del piano, o strutturali e, quindi, ingestibili. Occorre prendere coscienza ed esaltare il rilievo strategico degli eventuali punti di forza che l'impresa può giocare per il Piano di risanamento. Nella Fattibilità gestionale il valutatore/investitore ricerca una descrizione "veritiera" della situazione attuale della gestione, le strategie e le azioni da attuare per il risanamento, gli impatti occupazionali della riorganizzazione.

Nella Fattibilità economico-finanziaria occorre trovino spazio la misurazione delle perdite subite e l'analisi delle loro caratteristiche (perdite reddituali e perdite di valore economico) (tab. 2) ma soprattutto la riformulazione dei bilanci "inquinati" da isterismo da crisi (tab. 2). Una volta definite le azioni da intraprendere sarà vitale un'attenta analisi dei flussi di cassa previsti e dei fabbisogni finanziari necessari. In un contesto di tensione operativa e finanziaria, infatti, particolare attenzione va riposta nella gestione delle poste patrimoniali, sia attive che passive, al fine di non compromettere definitivamente $\mathrm{i}$ rapporti con gli stakeholders coinvolti, già duramente provati dalla ristrutturazione proposta, la cui collaborazione diventa essenziale per il buon esito del progetto.

Nell'ambito dell'analisi della Fattibilità economico-finanziaria è confermata l'importanza di alcune informazioni tipiche come il Fabbisogno finanziario necessario (tab. 2). In questa parte il Piano contiene indicazioni circa le strategie da adottare nella fase di emergenza e in quelle successive, con uno scheduling piuttosto puntuale: il tempo è importante! Le azioni da intraprendere da parte di un fondo dipendono dalla gravità della situazione: il fattore discriminante in tal senso è il cash flow.

Nella fase di emergenza, retrenchment, l'obiettivo dell'impresa è la sopravvivenza, ciò significa principalmente operare tagli per fermare l'emorragia di liquidità. Anche nel caso in cui la situazione non sia ancora gravissima, limitare le uscite di cassa è il primo modo per conservare risorse finanziarie da utilizzare per scopi che possano rendere l'impresa più competitiva. 
Tab. 2: Knowledge drivers in turnaround operations

\begin{tabular}{|c|c|c|}
\hline a) FATTIBILITA' & REDATTORE & INVESTITORE/VALUTATORE \\
\hline & $\begin{array}{l}\text { 1) Fattibilità gestionale } \\
\text { - Pianificare l'operazione } \\
\text { (piano di turnaround), } \\
\text { - Mercato, Settore } \\
\text { 2) Fattibilità } \\
\text { finanziaria. } \\
\text { - Opportunità di risanamento, } \\
\text { marginalità } \\
\text { - Indebitamento, } \\
\text { patrimonializzazione, } \\
\text { - Capitale economico stand } \\
\text { alone ex ante e Valutazione } \\
\text { ex post ans } \\
\text { - Timing analisi cash flow su } \\
\text { base trimestrale per } 1^{\circ} \text { anno } \\
\text { e poi annuale, -Analisi } \\
\text { sensitività } \\
\text { - Coerenza delle assumptions }\end{array}$ & $\begin{array}{l}\text { 3) Fattibilità gestionale } \\
\text { (piano di turnaround), } \\
\text { - I motivi della crisi e della situazione } \\
\text { attuale } \\
\text { - Le strategie e le azioni per } \\
\text { "cambiare" la situazione aziendale. } \\
\text { 4) Fattibilità economico-finanziaria. } \\
\text { - Riformulazione bilanci “inquinati” } \\
\text { - Misurazione Perdite } \\
\text { - Impatti occupazionali } \\
\text { - Costi della ristrutturazione } \\
\text { - Valore Capitale economico stand } \\
\text { - alone ex ante e ex post } \\
\text { - Timing analisi cash flow su base } \\
\text { mensile per } 1^{\circ} \text { anno e annuale per } 2^{\circ} \\
\text { e } 3^{\circ} \\
\text { - Analisi sensitività con wacc } \\
\text { - Exit strategy }\end{array}$ \\
\hline \multicolumn{3}{|l|}{ b) Risk analysis } \\
\hline Rischio di mercato & $\begin{array}{l}\text { 1)- Struttura del mercato: } \\
\text { - Barriere all'entrata } \\
\text { - Tasso di crescita } \\
\text { - Concentrazione } \\
\text { - Quota di mercato } \\
\text { - Fattori di vantaggio } \\
\text { competitivo dell'impresa }\end{array}$ & $\begin{array}{l}\text { 4)-Struttura del mercato } \\
\text { - Criticità delle barriere all'entrata } \\
\text { - Concentrazione concorrenza } \\
\text { - Struttura dei costi } \\
\text { - Quota di mercato }\end{array}$ \\
\hline $\begin{array}{l}\text { Rischio finanziario } \\
\text { ed economico }\end{array}$ & $\begin{array}{l}\text { 2) Autonomia finanziaria } \\
\text { - Indici di indebitamento } \\
\text { - Quoziente di disponibilità } \\
\text { - Leva finanziaria } \\
\text { - Free Operating Cash Flow } \\
\text { /Debito } \\
\text { - Leva operativa } \\
\text { - Indici di rotazione del capitale } \\
\text { circolante } \\
\text { - Ebitda/vendite }\end{array}$ & $\begin{array}{l}\text { 5) Indici di liquidità e solvibilità } \\
\text { - Indici di indebitamento } \\
\text { - Riserve/Mezzi Propri } \\
\text { - Leva finanziaria } \\
\text { - Posizione Finanziaria Netta/Ebitda } \\
\text { - Grado di leva operativa } \\
\text { - Indici di composizione } \\
\text { - Free Operating Cash Flow /Debito } \\
\text { - Indici di rotazione del capitale } \\
\text { - circolante } \\
\text { - Ebitda/vendite } \\
\text { - Roi, Ros, Investimenti aggiuntivi } \\
\text { (CapEx) } \\
\text { - Analisi di previsione del rischio di } \\
\text { default }\end{array}$ \\
\hline Rischio di progetto & $\begin{array}{l}\text { 3) Struttura finanziaria ex-post } \\
\text { NPV, IRR, BEP }\end{array}$ & $\begin{array}{l}\text { 6) Struttura finanziaria ex-post } \\
\text { IRR, BEP }\end{array}$ \\
\hline
\end{tabular}

Fonte: ns. elaborazione

Controllare il cash flow in timing mensile, per il fondo di turnaround, è decisivo (tab. 2). Occorre agire sui ricavi e sui costi da un lato e dall'altro sulle attività di bilancio: se queste ultime sono ridotte scende l'indebitamento e quindi il carico di oneri finanziari sul conto economico. Una strategia che raramente manca nella fase di retrenchment è la ristrutturazione del debito per ridurre il peso degli oneri finanziari sul singolo conto economico, ma soprattutto la riformulazione della gestione del circolante, per un più veloce smobilizzo delle partite. Come indicato 
nella tab. 2, sia il redattore che il valutatore hanno sottolineato l'importanza che il business plan presenti la valutazione del capitale economico stand alone ex ante il processo di turnaround e la stima del probabile valore ex post, con relativa analisi di sensitività sulla base degli elementi critici. L'analisi della Fattibilità economicofinanziaria è completata con lo sviluppo dei bilanci previsionali al fine di verificare gli effetti che gli interventi di risanamento potrebbero produrre. Il valutatore/investitore sarà, inoltre, interessato ad un holding period più contenuto, massimo 2 anni, entro il quale si attende di ritornare a produrre reddito e a valutare il way out. A corollario del ruolo comunicativo del Piano è necessario definire i sacrifici eventualmente da richiedere agli stakeholders e proiettare nel breve e nel medio termine i risultati ottenibili a seguito degli interventi e dei sacrifici richiesti, per valutare come potrà svilupparsi nel tempo l'eventuale ripresa.

La quantificazione del rischio di mercato è fondamentale, in entrambe le prospettive, al fine di individuare le forze negoziali in gioco e dove probabilmente si possono generare i margini. Un'informazione utile a tale scopo, sia per il redattore (tab. 2) che per l'investitore (tab. 2), è data dalle barriere all'entrata poiché, come già osservato, se basse, portano necessariamente ad una riduzione dei margini futuri. Altri aspetti basilari sono la quota di mercato detenuta e la struttura dei costi. Avere una posizione di leadership permette di ridurre il rischio delle proprie scelte, mentre guadagnare quote di mercato è particolarmente difficile e richiede l'implementazione di progetti innovativi ad incerto tasso di rientro. Particolare attenzione è, per questa fattispecie, l'analisi del rischio finanziario e quindi l'importanza informativa degli indici di liquidità, solidità e solvibilità. Nella logica del redattore (tab. 2), l'indice Riserve/Mezzi Propri può essere significativo poiché, se si prevedono un paio di anni di perdite, è importante avere riserve patrimoniali da destinare a copertura delle stesse. Entrambi gli operatori concordano nel segnalare che è il grado di leva finanziaria l'indicatore importante per il rischio finanziario: con l'indice Posizione finanziaria netta/EBITDA, difatti, è uno dei ratios che le banche utilizzano più spesso per confermare o revocare le linee di credito (tab. 2). Altri indici che si ritengono importanti per entrambe le figure di attori sono, FOCF/debiti, gli Indici di composizione, gli Indici di rotazione del circolante, in particolare il Costo del venduto sul magazzino, poiché un magazzino a veloce rotazione aumenta il rischio di obsolescenza delle rimanenze (tab. 2). Anche in queste fattispecie un approfondimento sul rischio di default mediante un'analisi del rischio d'insolvenza costituisce un must per l'investitore. L'indice EBITDA/Vendite ed il ROI sono significativi per rilevare il livello di marginalità dell'impresa. Riguardo al rischio finanziario si sottolinea che una struttura finanziaria equilibrata è il lavoro fondamentale del private equity. Anche l'IRR è un parametro fondamentale per l'investitore finanziario per l'analisi del rischio di progetto: è sulla base di questo che sono remunerati tutti gli stakeholders che richiedono ritorni piuttosto elevati, dato il rischio dell'investimento (tab. 2). Con il Break Even Point l'investitore riesce ad avere l'indicazione sulla difficoltà dell'obiettivo da raggiungere e a calibrare correttamente la struttura dei costi fissi. Le analisi di sensitività non sono reputate strettamente necessarie per un investitore in private 
equity, le variabili strategiche da sottoporre ad analisi what if sono comunque il fatturato, la marginalità operativa (EBITDA/Vendite), gli investimenti o l'andamento del capitale circolante netto.

\section{Conclusioni}

Come si è avuto modo di anticipare nelle parti precedenti, il business plan ha attraversato periodi di popolarità alternati a fasi in cui, soprattutto gli accademici, ne hanno messo in discussione la validità. Occorre ricordare che il business plan ha preso origine dal mondo operativo e che esso è considerato strumento di lavoro proprio dai practitioners. Le imprese più strutturate, di maggiori dimensioni e con management qualificato, in genere, hanno una procedura interna di Business plan Process. La questione si fa ancora più delicata, invece, per le piccole imprese che hanno bisogno, soprattutto nelle operazioni di expansion, di attivare nuovi canali di finanziamento o partnership. È nelle piccole realtà imprenditoriali che il business plan riveste la sua maggiore utilità, in considerazione del fatto che le nuove e/o piccole unità, che nel momento attuale costituiscono la parte più vivace del mercato, anche se più in difficoltà, si contraddistinguono per una cultura finanziaria e manageriale meno evoluta. Sono esse, però, che spesso promuovono progetti che investono settori ad alta turbolenza e quindi ad elevato rischio, specie nell'ambito del life science, dell'ITC, etc. Nonostante la sua importanza, il tema viene affrontato dagli operatori senza un framework teorico comune, universalmente accettato. Spesso, addirittura, nell'ambito del team di un singolo fondo coesistono approcci, metodologie e atteggiamenti sulla valutazione di un business plan, molto diversi tra loro, il che porta a discussioni non sempre efficaci. A differenza di altri settori della finanza, infatti, nel private equity esistono pochissimi strumenti teorici generalmente accettati: è un settore interamente basato sull'esperienza dei singoli, almeno a livello di chi lo pratica, e gli sforzi di sistematizzazione teorica rimangono confinati nelle Università. La letteratura si divide tra coloro che non riconoscono nessuna utilità del business plan per l'imprenditore, soprattutto se in fase costitutiva, e quelli che, invece, ne promuovono ancora la sua efficacia. Il mondo imprenditoriale in questa diatriba ha, comunque, ormai metabolizzato l'assioma che il business plan debba essere riconosciuto come conoscenza per supportare una consapevole formulazione delle strategie e come strumento per comunicare la conoscenza a tutti i players del mercato, con il quale si punta a legittimare all'esterno la volontà del business (Karlsoon e Honig, 2009). Anche se molti imprenditori sono apprensivi e titubanti nell'approccio al processo di business plan, considerandolo una fastidiosa incombenza, in molte occasioni si è avuto modo di sottolineare il fatto che i benefici realizzabili sono elevati (Arkenbauer 1995), soprattutto per quello che attiene la possibilità di individuare le opportunità ed identificare i problemi al fine di trovarne le possibili soluzioni, evitando che si generino reali conseguenze negative sulla gestione o sulla customer satisfaction (O'Connor 1998). Il business plan prima lo odi, poi lo apprezzi!!!! (Mariani, 2012a). Nella realtà, il processo di business plan è 
qualcosa di più di un documento, è la manifestazione di una strategia, con il quale si intraprende un percorso di conoscenza e di sintesi creativa che, prendendo avvio da una visione innovatrice e da alcune informazioni iniziali, riesce a plasmare la business idea, non immediatamente reale e realizzabile. I soggetti redattori, promotori dell'iniziativa, danno vita a un sistema dinamico, a un approccio permanente, che mette in relazione la visione con l'ambiente di riferimento e con le risorse necessarie. Si ricorda che le operazioni, soprattutto in caso di M\&A e special situations, si sviluppano in condizioni d'incertezza, per le quali progressivamente entrano in gioco strumenti più sofisticati per la valutazione. Partendo, difatti, dalla possibilità di identificare la visione aziendale per arrivare a definire le priorità strategiche, l'uso di un approccio Balance scorecard, consente di delineare le prospettive competitive cruciali per l'azienda ed i relativi fattori critici di successo; con l'introduzione progressiva di modelli stocastici i redattori possono progressivamente arricchire la capacità di pianificare e di monitorare le iniziative in condizioni di mercato incerte. Nella nostra analisi si è cercato di dare risposta alle esigenze di allineamento conoscitivo, in termini di Fattibilità dell'operazione e di livello di rischio, del redattore e del valutatore, evidenziando i knowledge drivers su cui i due players fanno leva. Da parte del fondo l'analisi del business plan rappresenta, difatti, un momento analitico fondamentale per dare alla decisione di investimento una base razionale coerente con il mandato fiduciario dato dai sottoscrittori del fondo. Come riportato da uno degli analisti che hanno partecipato all'indagine chi ben comincia è già a metà dell'opera. Non bisogna confondere questa finalità con una pretesa di indovinare esattamente il futuro - non è a questo che serve il business plan - ma piuttosto una dichiarazione programmatica circa le azioni strategiche da compiere in azienda. L'importante, difatti, è che il processo di planning sia dinamico, le strategie devono essere riformulate man mano che si arricchiscono le informazioni e gli output devono sempre essere rivisitati in ottica critica, evitando un'eccessiva "miopia emotiva". Occorre ricordare che il business plan non è solo un piano economico-finanziario dedicato a valutare la convenienza di un progetto, ma è il racconto del futuro di un'idea (Baccarani, 2009), dove le persone sono il punto centrale del successo. Nel discutere il business plan con il management che gestirà l'azienda dopo l'investimento, il fondo in effetti sta innanzitutto analizzando le persone, valutandone la visione strategica, la capacità di tradurre l'idea in assunzioni e decisioni coerenti, la loro disponibilità al confronto e alla condivisione del ragionamento in modo strutturato, la capacità di adattamento alle evoluzioni inaspettate che certamente ci saranno.

In definitiva, si investe sulle persone più che su uno specifico business plan, che però è un ottimo strumento per "conoscerle": il compito del redattore è quello di dare reale espressività ai molteplici numeri, importanti, ma anche volubili: in sostanza, un business plan è efficace se il redattore riesce a dar vita ai numeri! È su questo obiettivo strategico del processo di businees planning che si possono ancora scrivere interessanti riflessioni di tipo cross-culture, in grado di valorizzare le componenti antropologiche, per esaltare i risvolti strategici e di performance. 


\section{Bibliografia}

AGRAWAL A., JAFFE J. (2000), "The Post Merger Performance Puzzle", Advances in Mergers and Acquisitions, vol. 1, pp. 119-156.

AIFI PRICEWATERHOUSECOOPERS (2000), Guida pratica al capitale di rischio, Pubblicazione interna, Milano.

ALIPRANTIS C.D., CHAKRABARTI K., (2000), Games and Decision Making, Oxford University Press.

ALTMAN E. (2000), "Predicting financial distress of companies: revisiting the Z-score and Zeta models", in New York University Review, disponibile su http://www.stern.nyu.edu/, p. 22 e ss.

ALTMAN E.I., SABATO G. (2007), "Modeling credit risk for SMEs: evidence from the US market", «Abacus», vol. 43, n. 3, pp. 322-357.

ANDERSON T. (2000), "Build a Business Plan, research, buid contacts", in Business Journal, 08/18/2000, Kansas City.

ARKENBAUER J.B. (1995), Guide to Writing a High-Impact Business plan, McGraw-Hill, New York.

ARMSTRONG J. (1982), "The value of formal planning for strategic decisions: review of empirical research", Strategic Management Journal, vol. 3, n. 3, pp. 197-211.

ARNOLD, G. (2013), "Corporate financial management”, Fifth edition, Pearson, Chapter 20, pp. 849-902.

BACCARANI C. (2009), "Il business plan, la fiducia e il tempo degli altri”, Sinergie, numero speciale, giugno, pp. 7-14.

BAUM J. R., FRESE M., BARON R. A. (2007), The Psychology of Entrepreneurship, Lawrence Erlbaum Associations, Mahwah, New Jersey.

BEWAYO E.D (2010). "Pre-start-up Preparations: Why the Business planisn't always Written", The Entrepreneurial Executive, vol. 15, pp. 9-25.

BIGELLI M., MENGOLI S. (2004), "Private Benefits from New Acquisitions: Evidence from the Italian Stock Market", Journal of Management and Governance, vol. 8, n. 4, pp. 373-405.

BORGES C., HASHIMOTO M., LIMONGI R. (2013), “To plan or not to plan? An analysis of the impact of planning on the disbanding or growth of Brazilian start-ups, International", Journal of Entrepreneurship and Small Business, vol. 18, pp. 349-367.

BORELLO A. (2009), Il business plan: dalla valutazione dell'investimento alla valutazione del rischio, McGraw Hill, Milano.

BRANDIMARTE P., 2006, "Multi-Item Capacitated Lot-Sizing with Demand Uncertainty", in International Journal of Production Research, n. 44, pp. 2997-3022.

BRINCKMANN J., GRICHNIK D., KAPSA D. (2010), "Should entrepreneurs plan or just storm the castle? A meta-analysis on contextual factors impacting the business planning-performance relationship in small firms", Journal of Business Venturing, $\mathrm{n}$. 25, pp. 24-40.

BURKE A., FRASER S., GREENE F.J. (2010), "The Multiple Effects of Business planning on New Venture Performance", Journal of management studies, May, vol. 47, n. 3, pp. 391-416.

BRUNS V. (2004), "Who Receives Bank loans: A study of Lending officers' Assessments of Loans to growing small and medium sized enterprises", Jonkoping International Business School, Jonkoping.

CANDELO E. (2005), Le strategie di Turnaround, Tools, Egea, Milano. 
CARLESI A. (1999), Profili strutturali ed economico-finanziari delle imprese manifatturiere toscane, Franco Angeli, Milano.

CARLESI A., ANGELINI A., MARIANI G., BARONTINI R. (2002), Finanza per l'innovazione, Franco Angeli, Milano.

CARTWRIGHT S., SCHOENBERG R. (2006), "Thirty Years of Mergers and Acquisitions Research: Recent Advances and Future Opportunities", British Journal of Management, vol. 17, pp. S1-S5.

CASTROGIOVANNI G. (1996), "Pre startup planning and survival of new businesses: theoretical linkages", Journal of Management, vol. 22, n. 6, pp. 801-822.

CORREALE G., PENCO C. (2005),-"Business plan. Business as usual o qualcosa è cambiato?", Sistemi \& impresa, vol. 2, pp. 16-25.

CONCA V. ( 2010), Le acquisizioni, Egea, Milano.

D'ANNUNZIO N., FALAVIGNA G. (2004), Modelli di analisi e previsione del rischio di insolvenza: una prospettiva delle metodologie applicate, Torino, Ceris-CNR, Working Paper n. 17.

DATTA S., KODWANI D., VINEY H. (2013), "Shareholder wealth creation following M\&A: Evidence from European utility sectors", Applied Financial Economics, vol. 23, n. 10, May, pp. 891-900.

DELL'ANNO D., DEL GIUDICE M. (2002), "The spin-off model: a simultaneous way to knowledge transfer and entrepreneurship simulation", Proceedings "7th World Congress for Total Quality Management", Sinergie-Cueim.

DELL'ANNO D., VAN DER SIJEDE P., DEL GIUDICE M.A. (2004), "Descript-based approach to spin off: some first issues on innovative pathways of knowledge transfer and academic knowledge's reproducibility”, $12^{\circ}$ HTSF Conference, 25 May, Twenty University Press, The Netherlands.

DEPAMPHILIS D. (2012), Mergers, Acquisitions, and Other Restructuring Activities: An Integrated Approach to Process, Tools, Cases, and Solutions, Elsevier, Oxford.

DEUTSCH Y., KEIL T., LAAMANEN T. (2011), "A dual agency view of board compensation: the joint effects of outside director and CEO stock options on firm risk!", Strategic Management Journal, vol. 32, pp. 212-227.

FERRADINA A., CARRIERO F. (2010), Il Business plan, guida strategico operativa, III ed., Ipsoa, Milano.

FORD B.R., BORNSTEIN J.M., PRUITT P.T. (2008), Come si prepara un Business plan. La guida Ernst\&Young, III edizione, Tecniche Nuove, Milano.

GENDRON G. (2004), Forget the Elaborate Business plan, vol. 29, n. 10, pp. 87-88.

GOMES E., ANGWIN D.N., WEBER Y., TARBA S.Y. (2013), "Critical Success Factors through the Mergers and Acquisitions Process: Revealing Pre- and Post-M\&A Connections for Improved Performance”, Thunderbird International Business Review, vol. 55, n. 1 January/February, pp. 13-35.

GUATRI L. (1986), Crisi e risanamento delle imprese, Giuffrè, Milano.

GUATRI L. (1995), Turnaround, Declino, crisi e ritorno al valore, Egea, Milano.

GUMPERT D.E. (2002), Burn Your Business plan, Lauson Publishing, Needham.

HORMOZI A., SUTTON G., MINN M., LUCIO W. (2002),-’Business plans for new or small businesses: paving the path to success", Management Decision, vol. 40, n. 8, pp. 755-65.

HOING B. (2008), Institutional Isomorphism and the Likelihood of Business plan: The Moderating Effects of Environmental Uncertainty, Bobson Conference, USA.

HONIG B., KARLSOON (2004), "Institutional forces and written business plan", Journal of Management, vol. 30, n. 1, pp. 29-48. 
HOWELLS J.R.L. (2002), “Tacit Knowledge, Innovation and Economic Geography”, Urban Studies, vol. 39, n. 5-6, pp. 871-884.

HUI H., JING-JING Z. (2008). Relationship between corporate governance and financial distress: an empirical study of distressed companies in China", International Journal of Management voln. 25 pp. 32-38.

KATZ J.A. (2007), "Education and training in entrepreneurship", in Baum J.R., Frese M. Baron R., The psychology of entrepreneurship, Lawrence Earlbaum Associates, Inc., NJ.

KATZ J.A., GREEN R.P. (2007), Entrepreneurial Small Business, McGraw-Hill/Irwin.

KARLSOON T., HONIG B. (2009), "Judging a business by its cover: An Institutional perspective on new ventures and the business plan", Journal of Business Venturing, vol. 24, n. 1, pp. 27-45.

KING D., DALTON D., COVIN J., DAILY C. (2004), "Meta-Analyses of Post-Acquisition Performance: Indications of Unidentified Moderators", Strategic Management Journal, vol. 25 , n. 2, pp. 187-200.

KIRSCH D., GOLDFARB B., GERA A. (2009), "Form or substance: the role of business plan in venture capital decision making", Strategic Management Journal, vol. 30, pp. 487-515.

KUEHN K.W., GRIDER D., SELL R. (2009), "New Venture Assessment: Moving Beyond Business plans" in Introductory Entrepreneurship Courses in Journal of Entrpreneurship Education, vol. 12, pp. 25-42.

KWOKA J., POLLITT M. (2010). "Do mergers improve efficiency? Evidence from restructuring the US electric power sector", International Journal of Industrial Organization, vol. 28, n. 6, pp. 645-656.

LAABS J., SCHIERECK D. (2010), "The long-term success of M\&A in the automotive supply industry: determinants of capital market performance", Journal of Economics and Finance, vol. 34, n. 1, pp. 61-88.

LEE T.S., YEH Y.H. (2004). "Corporate Governance and Financial Distress: evidence from Taiwan", Corporate Governance, vol. 12, n. 3, pp. 378-388.

LEWITT B., MARCH J.G. (1988), “Organisational learning”, Annual Review of Sociology 14, pp. 319-340.

LUMPKIN T.G., SHRADER R., HILLS G.E. (1998), "Does formal business planning enhance the performance of new ventures", in Reynolds PD., Frontiers of Entrepreneurship Research, Pand Pubblication, Babson Park.

MARAFIOTI E. (2005). "Consolidare la crescita internazionale: il contributo di alleanze ed acquisizioni", Economia \& Management, vol. 5, pp. 71-87.

MARIANI G. (2002), "Il business plan come strumento di valutazione degli investimenti e di pianificazione dei fabbisogni", in Carlesi A. (a cura di), Finanza per l'innovazione. Esperienze didattiche-scientifiche all'interno del Progetto Link, Franco Angeli, Milano.

MARIANI G., MARSILI V. (2011), "The Corporate Governance in Turnaround strategy: the definition of index of good governance and evidence on performance", GSTF Business Review, vol. 1, n. 1, pp. 150-155.

MARIANI G. (2012a), Conoscenza e creazione di valore. Il ruolo del business plan, Franco Angeli, Milano.

MARIANI G. (2012b), (a cura di), Dalla crisi alla creazione di valore: il processo di Turnaround, Pisa University Press, Pisa.

MARIANI G., PANARO D. (2012), "Corporate Governance and Performance in turnaround: a Synthetic Index" in Corporate Ownership \& Control, vol. 10, pp. 62-74. 
MORRISETTE S., HATFIELD L. (2010), "Second draft of a business plan: What should it contain?", Journal of the International Academy for Case Study, vol. 16, n. 2, pp. 5265 .

MARTYNOVA M., RENNEBOOG L. (2009), What determines the financing decision in corporate takeovers: Cost of capital, agency problems, or the means of payment?, Journal of Corporate Finance, vol.15, pp. 290-315.

MULLINS R. (1999), "Business plans are critical for small companies", Business Journal Serving Greater Milwaukee, 12/19/99 pp. 101-121.

NETTER J., STEGEMOLLERB M., WINTOKIC M.B. (2010), "Implications of data screens on merger and acquisition analysis: A large sample study of mergers and acquisitions from 1992-2009", SSRN, November 2, 2010, http://ssrn.com/abstract=1510724.

O'CONNOR T. (1998), "Take the initiative to write a viable business plan", Denver Business Journal, 02/09, February, p.21A.

OECD (1996), Employment and Growth in the Knowledge-Based Economy, OECD, Paris.

PAROLINI C. (2011), Business planning, Pearson, Milano.

PERRY C.S. (2001), "The Relationship between Written Business plans and the Failure of Small Business in THE U.S.”, Journal Of Small Business Management, vol. ???? n. 39, pp. 109-135.

PIANA M. (2012), "Business plan: il punto di vista di un investitore finanziario", in Mariani G., Conoscenza e creazione di Valore, Franco Angeli, Milano.

PINCH S., HENRY JENKINS M., TALLMAN S. (2003), "From 'Industrial District' to Knowledge Clusters': A Model of Knowledge Dissemination and Competitive Advantage in Industrial Agglomerations", Journal of Economic Geography, vol. 3, pp. 373-388.

PODDIGHE F., MADONNA S. (2006), (a cura di), I modelli di previsione delle crisi aziendali: possibilità e limiti, Giuffrè, Milano.

ROJAS-ARCE J., GELMAN O., SUAREZ-ROCHA J. (2012), "The methodology for strategic plan implementation", Journal of Applied Research and Technology, Vol. 10 No.2, April, pp. 248-261, Mexico City.

ROSS L.M. (2000), "Businessplan.com", The Oasis Press, Oregon.

SADARSANAM P.S. (2003), Creating Value from Mergers and Acquisitions, Prentice Hall, London.

SAHALMN W. (2007), "How to write a great business plan", Harward Business Rewiew, Jul-Aug, pp. 98-108.

SARASVATHY S.D. (2001), "Causation and effectuation: toward a theoretical shift from economic inevitability to entrepreneurial contingency", Academy of Management Review, vol. 26, n. 2, pp. 243-263

SCHAEFER P. (2011), "The Seven Pitfalls of Business Failure And How to Avoid Them", Attard Communications, www.businessknowhow.com/startup/business-failure.htm.

SEGATO G., MANIERO K. (2001), "Operazioni di finanza straordinaria e business planning", Amministrazione e Finanza Oro, vol. 12, n. 4, pp. 49-75.

SEVERINO M. (2009), "Strategie e modalità operative degli investitori in capitale di rischio che lavorano con le start-up", Innov'azione, 29 aprile 2009, vol. 4, pp. 15-32.

SIMONE M. (2006), "Start - up aziendale: costruzione del Business plan e ricerca dei finanziatori”, Amministrazione e finanza, vol. 18, n. 24, pp. 40-45.

STUTELY R. (2008), Il Business plan, seconda edizione, Pearson.

SUDARSANAM S. (2003). Creating value from mergers and acquisitions: The challenges: An integrated and international perspective. London: Financial Times-Prentice Hall. 
THOMSEN M. (2009), The Dynamic Business plan, Thomsen Business Information, Hojbjerg.

TIMMONS J., SPINELLI S. (2007), New Venture Creation: Entrepreneurship for the 21st Century $\left(7^{\circ}\right.$ Ed.), McGraw-Hill/Irwin, NY

ULIJN M.J., DUILL M.O., ROBERTSON S. (2004), “Teaching Business plan Negotation”, Business Communication Quarterly, vol. 67, n. 1, pp. 41-57.

WAN W.P., YIU D. (2009), From Crisis To Opportunity: Environmental Jolt, Corporate Acquisitions, And Firm Performance, Strategic Management Journal, vol. 30, pp. 791-801.

WEBER Y., RACHMAN-MOORE D., TARBA S.Y. (2012), "HR practices during postmerger conflict and merger performance", International Journal of Cross Cultural Management, vol. 12, n. 1, April, pp. 73-99.

ZANETTI L. (2000), La valutazione delle acquisizioni, Egea, Milano. 
